\title{
Case analysis of integrated maintenance technology for multi- dimensional rapid detection and trenchless reinforcement
}

\author{
He Yunwu ${ }^{2}$, Liu Tao ${ }^{1}$, Wang Tao ${ }^{1}$, Liang Xiayi ${ }^{1}$, Wei Hanxin ${ }^{1}$, Zheng Zhigang $^{1}$, Xiao Xin ${ }^{3 *}$ \\ ${ }^{1}$ Shenzhen Yuetong Construction Engineering Co., Ltd, 518019, Shenzhen, China \\ ${ }^{2}$ Shenzhen Tagen< group $>$ Co., Ltd, 518034, Shenzhen, China \\ ${ }^{3}$ Foshan University, 528000, Foshan, China
}

\begin{abstract}
China's highway construction has moved from "construction-oriented" to the development stage of "equal attention to construction and maintenance". The infrastructure represented by urban expressways, under heavy and complex traffic loads, is prone to a variety of diseases that take place in the middle and lower layers of pavement such as "frost boils" and "voids", which cannot be repaired ideally with the help of conventional detection and treatment techniques. In order to solve the above problems, this paper adopts nondestructive testing plans such as ground penetrating radar and falling weight deflectometer to conduct multidimensional rapid detection of the road surface to obtain the image information and mechanical data of the road structure. Based on the improved calculation method, the pavement disease area, depth and type can be effectively judged. Combined with the observation of water level, the polymer grouting reinforcement plan is designed to eliminate the problems in the middle and lower layers of the road surface. It can effectively reduce the incidence of diseases in the upper layer of the sidewalk, and significantly improve the efficiency and service level of the sidewalk.
\end{abstract}

\section{Introduction}

With the development of the economy, China's highway construction has moved from "construction-oriented" to the development stage of "equal attention to construction and maintenance". It's also necessary to improve and upgrade the road surface detection and maintenance technologies accordingly, in particular for a variety of diseases including "frost boils" and "voids". These diseases are difficult to detect as they occur in the middle and lower layers of the pavement and cannot be repaired accurately by conventional methods. These diseases are difficulties in terms of road maintenance, which seriously affects road traffic efficiency and service level.

In recent years, Ground Penetrating Radar technology and Falling Weight Deflectometer technology are two mainstream technologies for accurately identifying diseases in the middle and lower layers of the road surface under non-destructive conditions. The mechanism of Ground Penetrating Radar (GPR) is to excite highfrequency electromagnetic waves through the ground penetrating radar antenna, propagate to the road structure, and produce reflections on the contact surface with different dielectric characteristics, which are recorded by the receiving antenna. The internal radar images of the road are obtained through a series of processing methods such as denoising, filtering, component model, etc. ${ }^{[1-2]}$. Featuring non-destructive, fast, perspective, continuous and high-precision, the GPR technology is gradually applied in the field of road surface detection and maintenance and it can better detect the detailed information of hidden diseases of the road structure such as cracks, voids, uneven settlement, abnormal moisture content ${ }^{[3-5]}$, the theoretical foundation of which lies in that electromagnetic wave reflections occur at the medium interface of different dielectric properties, which have experienced unloaded pulses, high-speed pulses, highfrequency pulsed electromagnetic waves and other technical systems ${ }^{[6-9]}$.

Falling Weight Deflectometer (FWD) is a pulsepowered deflection meter produced in the early 1970 s and is another important method for non-constructive identification of road diseases. It has the advantages of no damage, fast speed, high accuracy, etc., and it simulates the effect of driving load well. The detection result is the deflection basin data, so it is increasingly widely used all over the world. It is mainly used in pavement maintenance management ${ }^{[10]}$.

Polyurethane material is a polymer with dual properties of metal and rubber (ie. Toughness and elasticity). It is mainly produced by the reaction of isocyanate and polyol. Containing no less than two isocyanate and hydroxyl functional groups, its properties are mainly determined by the isocyanates, polyols, and the additives of the synthesis process. It were the German scholars that first discovered the method to obtain polyurethane through polymerization ${ }^{[11]}$. It is extensively used in elastomers, coatings, rigid plastic foams and adhesives. After 1970, polymer grouts have gradually been applied to the foundation reinforcement of industrial

* Corresponding author: 604849392@qq.com 
and civil buildings, foundation subsidence elevation, airport runways, workshop floors, slab bottom voids, slab bottom elevation and other locations.

In this study, the maintenance of a certain road section of the eastbound section of Shenzhen Nanping Express Phase I was taken as an example to study multidimensional rapid detection and trenchless reinforcement technology. Confined by the construction conditions of the road section and the site environment as it is located at the bottom of the mountain slopes and valleys on both sides, the method of dumping stones to squeeze the silt and filling was used. After a period of operation, diseases such as frost boils, mud, potholes occur all the year round on some lanes for heavy vehicles. The effects of multiple repairs using the conventional methods are not satisfactory and the hidden dangers still remain. This research first used GPR and FWD to determine the area, depth and type of the diseases on the road section; then, it used the polymer grouting technology to continue to repair the underlying structure of the road surface; finally, the ground penetrating radar was used to comprehensively evaluate the curing effect of polymer grouts.

\section{Multi-dimensional rapid detection method}

\subsection{Ground penetrating radar technology}

Ground Penetrating Radar (GPR) works on the principle that when high frequency electromagnetic waves are excited by the ground penetrating radar antenna and incident vertically onto a multi-layer pavement structure, transmission and reflection phenomena will occur at the interface of different media, and the transmission and reflection coefficients are as follows.

$$
\begin{aligned}
& Z(n)=\frac{2 k_{n}}{k_{n}+k_{n+1}} \\
& R(n)=\frac{k_{n}-k_{n-1}}{k_{n}+k_{n+1}}
\end{aligned}
$$

where, $R$ (n) and $Z$ (n) denotes the transmission coefficient and the reflection coefficient when the electromagnetic wave reaches the $\mathrm{n}^{\text {th }}$ layer and the $\mathrm{n}+1^{\text {th }}$ layer. $K_{n}$ and $K_{n+1}$ are the propagation constants of electromagnetic waves in the $\mathrm{n}^{\text {th }}$ and $\mathrm{n}+1^{\text {th }}$ layers of the medium, respectively. By calculating the amplitude of each transmitted and reflected wave, the propagation time of electromagnetic waves in each structural layer can then be clarified.

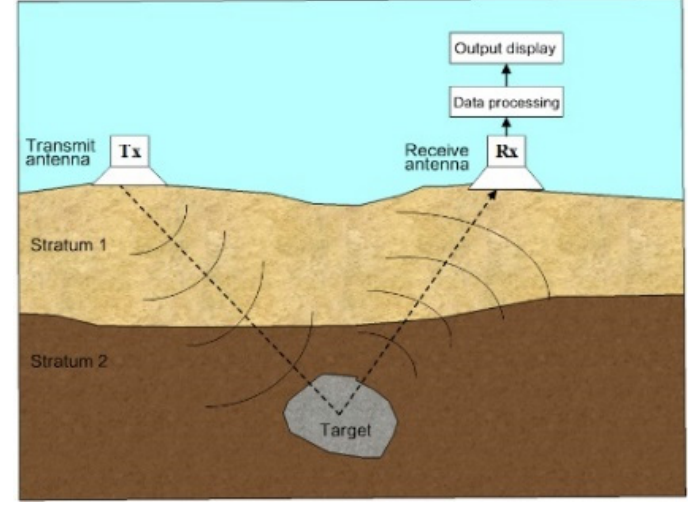

Fig.1 Ground penetrating radar detection schematic

Take the air-coupled antenna as an example. The distance between the transmitting antenna and the receiving antenna is very small (double-base antenna) or combined into one (single-base antenna). When the bottom inclination angle is small, the incident path of electromagnetic waves can be considered perpendicular to the road surface. The incident angle and the refraction angle of the electromagnetic wave can be assumed to be 0 . With the imaginary part of the dielectric constant of the material not considered, the road thickness calculation formula can be:

$$
h_{1}=\frac{c}{2 \sqrt{\varepsilon_{1}}} \Delta t_{1}
$$

The propagation of electromagnetic waves emitted by ground penetrating radar in the pavement structure will be affected by various factors and different structural materials have different attenuation effects on the electromagnetic waves. Thus, it is necessary to adopt a modified equation to estimate the detection depth, as follows:

$$
\frac{p_{r \min }}{p_{t \max }}=\frac{\eta_{T X} \eta_{R X} G_{T X} G_{R X} \lambda^{2} m \sigma_{b} e^{-4 \alpha d_{\max }}}{64 \pi^{3} d_{\text {max }}^{4}}
$$

The minimum detectable signal power of the ground penetrating radar is $\mathrm{P}_{\mathrm{rmin}}=\mathrm{kT}_{\mathrm{n}} \mathrm{B}_{\mathrm{n}} \mathrm{F}_{\mathrm{n}}(\mathrm{S} / \mathrm{N})$ min, and the maximum transmit power $\mathrm{P}_{\text {rmin }}$. The Boltzmann constant is $\mathrm{k}$, the equivalent noise temperature of the receiving unit is $T_{n}$, the noise bandwidth is $B_{n}$, the noise index is $F_{n}$, the scattering cross-sectional area of the target is $\sigma_{\mathrm{b}}$, and the gains of the ground penetrating radar transmitting antenna and receiving antenna are $\eta_{\mathrm{Tx}}$ and $\eta_{\mathrm{R}} \mathrm{x}$, the wavelength of the electromagnetic wave is $\lambda$, and the maximum detection distance of the ground penetrating radar is $d_{\max }$.

According to the electromagnetic theory, the wavelength $\lambda_{m}$ of electromagnetic waves in structural materials is:

$$
\lambda_{m}=\frac{c}{f_{c} \sqrt{\varepsilon_{r} \mu_{r}}}
$$

Where, $\mathrm{f}_{\mathrm{c}}$ denotes the center frequency of the pulse signal, $c$ represents the propagation speed of electromagnetic waves in vacuum, $\mathrm{u}_{\mathrm{r}}$ and $\mu_{\mathrm{r}}$ and $\mathrm{gr}$ are the permeability and relative permittivity of the structural material, respectively. The greater the relative permittivity and the permeability of the structural material, the higher 
the center frequency of the antenna, and the smaller the maximum depth that the ground penetrating radar can detect. The relationship between the antenna frequency and the detection depth is shown in Table 1.

Table 1 Detection depth values of antennas with different frequencies

\begin{tabular}{|l|l|}
\hline Frequency & Depth value \\
\hline $2.5 \mathrm{GHz}$ & $30 \mathrm{~cm}-60 \mathrm{~cm}$ \\
\hline $1.0 \mathrm{GHz}$ & $60 \mathrm{~cm}-1.0 \mathrm{~m}$ \\
\hline $900 \mathrm{MHz}$ & $75 \mathrm{~cm}-1.5 \mathrm{~m}$ \\
\hline $500 \mathrm{MHz}$ & $1.5 \mathrm{~m}-3 \mathrm{~m}$ \\
\hline $300 \mathrm{MHz}$ & $3 \mathrm{~m}-6 \mathrm{~m}$ \\
\hline $100 \mathrm{MHz}$ & $10 \mathrm{~m}-20 \mathrm{~m}$ \\
\hline
\end{tabular}

\subsection{Falling Weight Deflectometer}

Road surface deflection refers to the total vertical deformation (total deflection) or vertical rebound deformation value (rebound deflection) produced by the surface wheel gap position of the subgrade pavement under the specified standard axle load, and the unit is $0.01 \mathrm{~mm}$, It can effectively reflect the bearing capacity of the pavement structure, that is, the cumulative number of driving loads and the service life that can be withstood before the predetermined damage condition is reached. Based on the test result of the deflection $\left(D_{1}\right)$ of the center point, the pavement structure strength coefficient (SSI) is obtained. Convert it into a percentile system and adopt the pavement structure strength index (PSSI) as the evaluation index.

$$
\begin{gathered}
\text { PSSI }=\frac{100}{1+\mathrm{a}_{0} \mathrm{e}^{\mathrm{a}_{1} S S \mathrm{i}}} \\
S S I=\frac{l_{d}}{l_{0}}
\end{gathered}
$$

where SSI denotes the pavement structure strength coefficient; $l_{d}$ represents the design deflection of the pavement, $\mathrm{mm} ; l_{0}$ is the measured deflection, $\mathrm{mm} ; a_{0}$ is the model parameter, which is $15.71 ; a_{1}$ is a model parameter, which is -5.19 .

The falling weight deflectometer used in this paper is a Dynatest 8000 FWD. The hydraulic system is controlled by a computer to lift and drop the weight to subject the pavement surface to a load pulse. The load is adjusted by changing the weight and the lifting height. The load range is 7 to $120 \mathrm{KN}$, the resolution of the deflection sensor $1 \mu \mathrm{m}$, the system error is $\pm 2 \%$, the number of deflection sensors 9 , and the diameter of the load plate $30 \mathrm{~cm}$, which can reflect the mechanical information of the road structure to a certain extent. The measurement speed is fast, and the deformation recognition accuracy is high. It can effectively simulate the dynamic effects of driving load, and through multi-point deflection measurement, it lays the foundation for the inversion of pavement structure. It is the standard equipment for long-term tracking observation and service performance research of the US Strategic Highway Research Program (SHRP) pavement.

Table 2 Layout of Falling Weight Deflectometer Sensor

\begin{tabular}{|l|l|l|l|l|l|l|l|l|l|}
\hline Sensor No. & \multicolumn{1}{|l}{ 1\# } & $2 \#$ & $3 \#$ & $4 \#$ & $5 \#$ & $6 \#$ & $7 \#$ & $8 \#$ & $9 \#$ \\
\hline & & & & & & & & & \\
Distance from the load center (mm) & 0 & 200 & 300 & 500 & 600 & 900 & 1200 & 1500 & 1800 \\
\hline
\end{tabular}

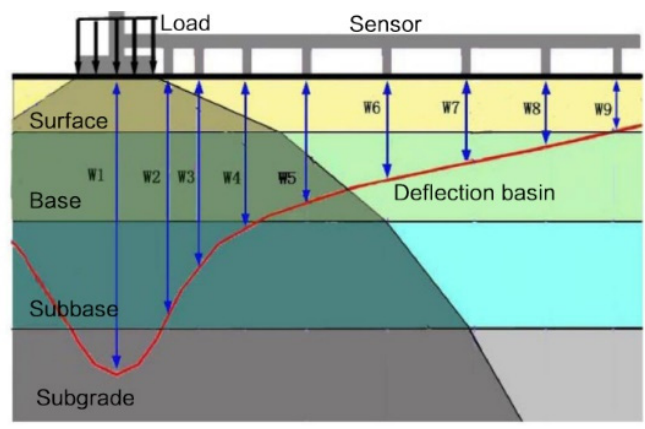

Fig. 2 The working principle of the falling weight deflectometer

\section{Trenchless reinforcement maintenance technology}

The semi-rigid base layer diseases are located under the pavement, which is difficult to identify and treat. They often cause serious diseases such as frost boils and potholes on the surface of the pavement. Treatment such as additional paving, excavation for repair and joint sealing cannot root out the structural damage problems. The polymer grouting technology is used in this paper to treat hidden diseases in the middle and lower layers of heavy-duty traffic pavement. Studies have shown that polymer grouting materials feature good rheological properties and the loss modulus of the material is much greater than the storage modulus. And the phase angle is also both are greater than $45^{\circ}$. Viscous deformation often occurs under the action of alternating stress. Certain structural strength can be formed shortly after the material is injected into the diseased position of the semi-rigid base layer, playing a role in supporting its own structure, as shown in Fig. 3. Its viscosity increases rapidly with the increase of the temperature and the curing time is accelerated. Therefore, during grouting, the heating temperature of the equipment can be changed to respond to different temperature construction environments to ensure material performance. 


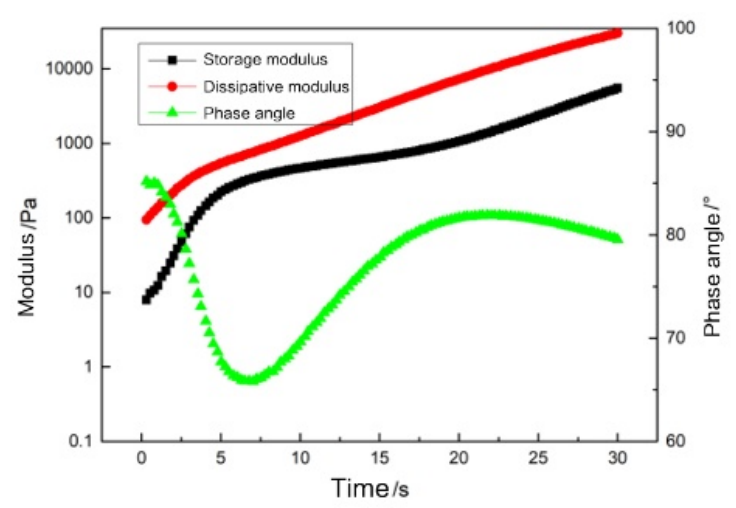

Fig. 3 The rheological properties of the polymer material

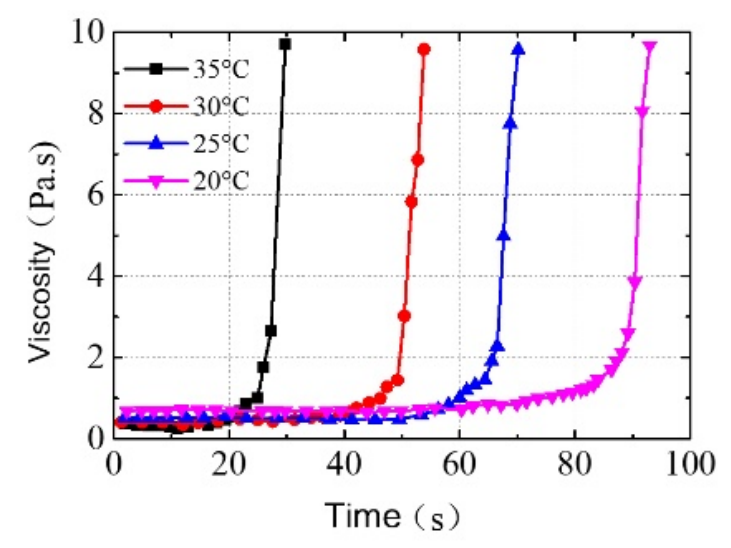

Fig. 4 Change curves of viscosity of polymer materials with time at different temperatures

The viscosity of the polymer grouting material increases rapidly with the increase of temperature and the setting and curing time is accelerated. The heating temperature of the grouting equipment can also be changed to respond to construction environments of different temperatures to ensure the material properties. In the meantime, the polymer grout features apparent expansibility. Take a fixed container volume test of polymer material with a density of $0.18 \mathrm{~g} / \mathrm{cm}^{3}$ for instance. There are three expansion phases. The first phase is when the container is filled, which is within 20 s after the start of grouting. As the grout hasn't fully filled the container, the result of the measured expansion force is $0 \mathrm{MPa}$. In the second phase, the expansion force increases rapidly with time and can rise up to more than $80 \%$ of the maximum value. And the time range is 20 to $40 \mathrm{~s}$, during which time, the container is fully filled with grout material but the chemical reaction is still in place. The material volume expands rapidly. Confined by the container volume, the material expansion force skyrockets. And the grout material density increases with it. In the final phase, the expansion force increases slowly with time. The action time range lasts from $60 \mathrm{~s}$ to the end of the reaction, indicating a slow chemical reaction inside the material.

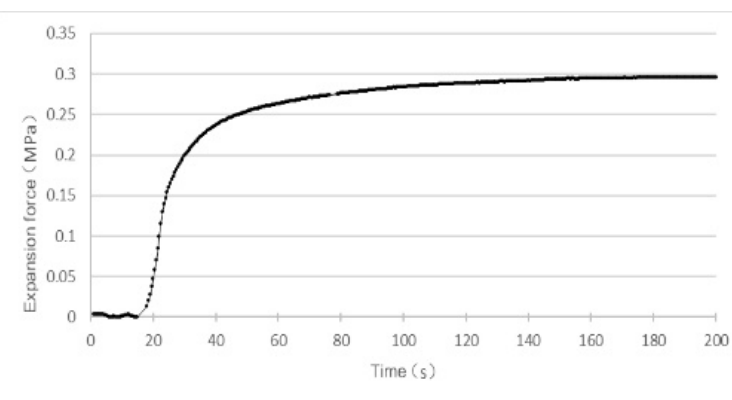

Fig. 5 Changes of the expansion force of the polymer material (density: $0.18 \mathrm{~g} / \mathrm{cm}^{3}$ )

To sum it up, polymer material feature good rheology, expansibility and toughness, and can coordinate well with the rock and soil. Thanks to a light density, no additional load is generated on the structure. $0.5 \mathrm{MPa}$ to $10 \mathrm{MPa}$ expansion force can be produced in the quick reaction, which makes it quickly to fill the voids as it is injected into the specific position of the semi-rigid base, thereby lifting the compacted soil and draining the underlying water. It permeates into the voids and cement the base material and enhance the pavement strength.

\section{Engineering application case analysis}

\subsection{Project Overview}

Since Nanping expressway was open to traffic in 2006, it has significantly eased the traffic pressure in the center of city center. Diseases such as severe water penetration, frost boils, potholes occur on some of the pavement due to the repeated action of heavy-duty traffic, which cannot be fundamentally solved using conventional maintenance measures.

The on-site investigation and the construction record investigation shows that Nanping expressway locates at the valley sandwiched by mountains. It is a filled section and its subgrade is formed by dumping stones and squeezing silt. And the subgrade construction quality is not good. Under the action of vehicle load and rainwater penetration, uneven settlement occurs and leads to loose areas of subgrade, causing pavement diseases.

\subsection{Multi-dimensional rapid detection plan design}

(1) Ground penetrating radar detection plan design

In this paper, the SIR-30E ground penetrating radar developed by American GSSI company was used. It is composed of a control host, an antenna, and a notebook computer connector. It features a transceiver integrated antenna, a frequency of $400 \mathrm{MHz}$, a continuous acquisition method, a fixed-point mileage wheel, and a sampling interval of $2 \mathrm{~cm}$. The acquisition time window is $35 \mathrm{~ns}$ and uses exponential gain mode.

The radar detection profile was arranged along the driving direction of the test section, and 2 survey lines (left and right wheel tracks) were arranged in each lane, and the survey lines were appropriately densified according to the actual situation on the spot to accurately locate the 
diseased area.

The data collected by the radar were processed by zero point correction, profile distance correction and gain adjustment. Then the in-phase axis was formed according to the radar waveform. The human-computer interaction mode was used to inteprete the information so as to outline the defects in the each structural layer of the road surface and the middle and lower layers of the road surface.

(2) Design of the detection plan of the falling weight deflectometer

This paper conducted deflection detection on the east section of Nanping Expressway (total length of 100 meters). The detection marked its corresponding measuring points and the load was set to be 5T. The position of the measuring point was set to be 5 meters from the right wheel tracks of the lane.

\subsection{Trenchless reinforcement scheme design}

Different grouting voids were arranged for different types of pavement diseases. The diameter of the grouting hole was set to be $0.016 \mathrm{~m}$. The holes were drilled to the top of the subgrade. The length of the grouting pipe was decided according to the grouting repair requirements. The reinforcement was arranged for the entire lane. The holes were arranged to be $0.6 \mathrm{~m}, 1.5 \mathrm{~m}$ and $1.5 \mathrm{~m}$ one by one from the shoulder of pavement to the center of the road. And the holes were also arranged every $1 \mathrm{~m}$ from the longitudinal section of the lane, as shown in Fig. 6.

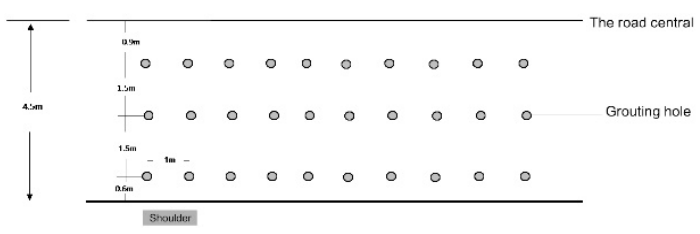

Fig. 6 Schematic of grouting hole distribution

The specific process is as follows: First, a percussion drill was adopted to drill holes to designed depth at the marked position and the road was timely cleaned so there was no pollution. Second, the grouting iron pipe was cut into different lengths and was introduced through the grouting holes. Again, the concave edge of the injection cap was cleaned using a special tool so it can better integrate with the injection gun. The cleaned up injection cap was installed to the grouting pipe end. Finally, the amount grout was determined according to the disease require requirement and the injection gun and the injection cap were fastened using the fixture to provide fixed pressure for the equipment. A and B polymer material were transferred to the injection gun through the conveying pipeline. The two materials were injected to the pavement diseases areas through the grouting tube at the injection muzzle and chemical reactions occurred. The liquid material solidified with rapid volume expansion to repair the diseases.

\subsection{Grouting reinforcement performance evaluation}

(1) Evaluation of the ground penetrating radar performance

Different structural interfaces of the pavement can be basically determined according to the profiles provided by the ground penetrating radar. The position of the disease areas can be detected to compare with the images before and after the grouting. The horizontal direction is the distance from the test starting point and the vertical direction is the detection depth.

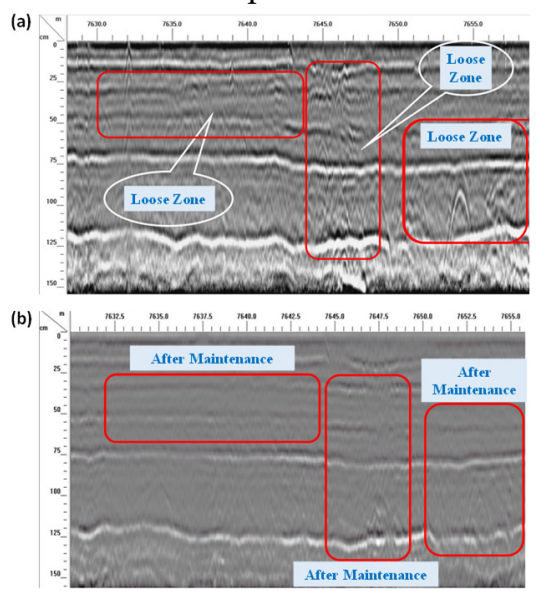

Fig.7 K7+630-k7+655 road left line GPR image
(a) Before repair, (b) After repair

The GPR image of the left line of the K7+630-K7+655 section is shown in Fig. 8(a). It can be seen from the figure that there was a problem of uneven settlement under the action of the vehicle before grouting and curing, and there were obvious reflective interfaces and obvious loose areas, especially the loose areas from the subgrade to the road surface at the point of $\mathrm{K} 7+645$. It can be seen from Fig. 8(b) that after grouting reinforcement treatment of the middle and lower diseases, the reflected signal amplitude of the radar image is small and the image shows balance and the reflection interface is not obvious, indicating that the loose areas with high water content were filled and compacted with polymer material and it presents good reinforcement effect.

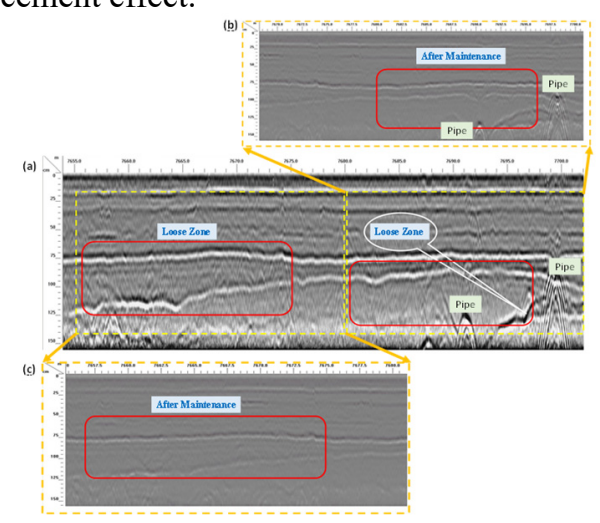

Fig. 8 GPR image of the left lane of the K7+655-k7+700

Fig. 8 is the GPR image of the left line of the K7+655$\mathrm{k} 7+700$ section, where it can be seen that loose areas with 
high water content existed in $\mathrm{K} 7+655-\mathrm{K} 7+655$ before repair. Confined by two pipelines, the compactness was not sufficient between the two pipelines close to K7+690. After grouting reinforcement, it can be seen from Fig. 8(b) and (c) that the refection cross area is no longer obvious and the image is more even, indicating that under the extrusion action of the polymer, the loose areas with high water content have been compacted, indicating good reinforcement effect.

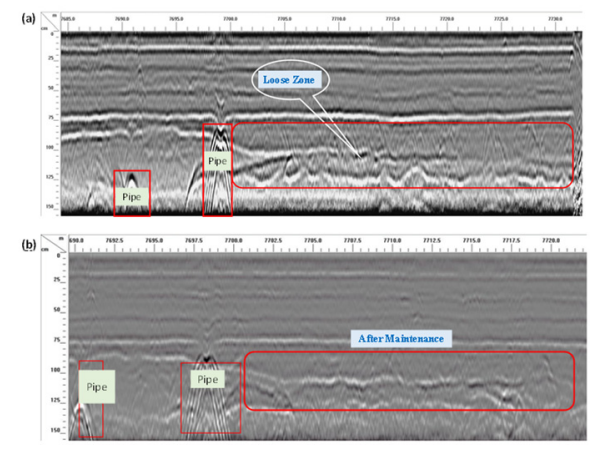

Fig. 9 GPR image of the right line of the K6+690-K7+720 section

(a) Before repair, (b) After repair

It can be seen from Fig.10 that the GPR image of the right line of $\mathrm{K} 6+690-\mathrm{K} 7+720$ is similar to that of the left line and loose areas existed in $\mathrm{K} 7+700-\mathrm{K} 7+720$ area. Fig.9 (b) shows that after grouting the image presents less obvious reflection profile and the image is more even indicating that the loose areas with high water content has been compacted under the extrusion action of the polymer, achieving good reinforcement effect.

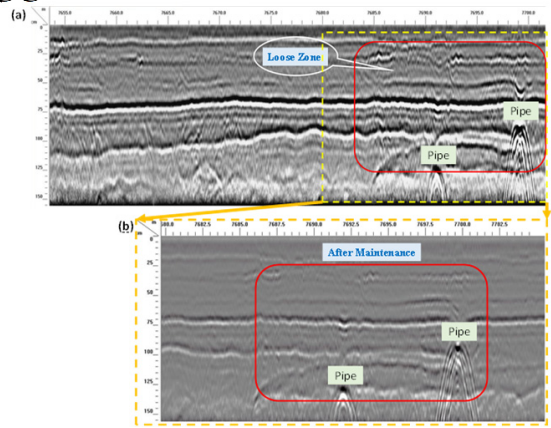

Fig. 10 GPR image of the right line of the K $7+655-K 7+700$ section

(a) Before repair, (b) After repair

It can be seen from Fig. 10 that there existed serious loose areas with high water content in the road structure above the pipeline of the $\mathrm{K} 7+655-\mathrm{K} 7+700$ GPR image. After grouting, it can be seen from Fig. 10(b) that the loose areas with high water content have been compacted under the extrusion action of polymer, indicating that polymer grout can achieve good repair and maintenance effect for the loose areas nearby the pipelines.

(2) Analysis of the detection effect of the falling weight deflectometer

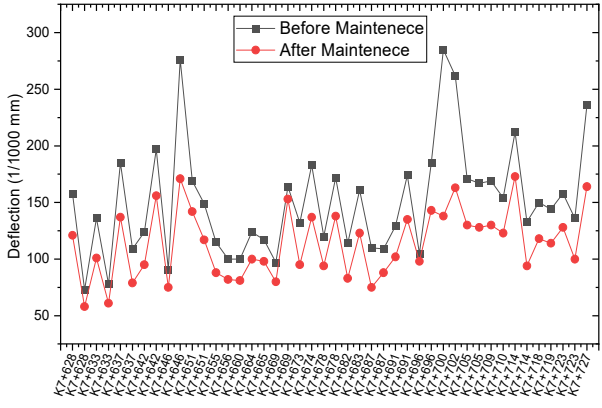

Fig. 11 Comparison of deflection before and after grouting

It can be seen from the test results of the falling weight deflectometer in Fig. 11 the deflection value of the road surface before grouting reinforcement was $285 \mu \mathrm{m}$ at the maximum, $73 \mu \mathrm{m}$ at the minimum, and the average value was $151 \mu \mathrm{m} .24 \mathrm{~h}$ after grouting, the maximum measured deflection value was $173 \mu \mathrm{m}$, the minimum was $58 \mu \mathrm{m}$, the average value was $114 \mu \mathrm{m}$, and the average drop was $30 \mu \mathrm{m}$, which satisfied the design deflection with an average drop of $20 \%$, presenting good grouting reinforcement effect.

The comparison of the test results of the falling weight deflectometer and the ground penetrating radar shows that before grouting, the deflection of the K7+700 and $\mathrm{K}+642$ reached the highest value indicating that that section was subject to the most loose situation. It can be seen from Fig.7 and Fig. 11 that the maximum deflection value corresponds with the severely loose area in the GPR image, indicating that the detection results of the falling weight deflectometer and the grounding penetrating radar are highly consistent.

\section{Conclusion}

(1) Ground penetrating radar technology can effectively identify hidden diseases in the middle and lower layers of heavy-duty traffic roads. Combined with daily detections and historical data, it can roughly determine diseases information such as the scope and depth and provide corresponding technical basis for grouting reinforcement .

(2) The falling weight deflectometer can provide a specific basis for judging the stress performance of heavyduty traffic roads, and the results are highly consistent with GPR images, which effectively improves the accuracy of the road health evaluation.

(3) It can be seen from the results of ground penetrating radar and falling weight deflectometer that the use of polymer grouting technology can squeeze the loose areas with high water content and fill and compact the voids to solve the diseases such as "frost boils" that are difficult to treat at a relatively ideal price. It has good economic and social benefits.

\section{References}

1. Lahouar, S.; Al-Qadi, I.L. Automatic detection of multiple pavement layers from GPR data. NDT \& E International $2008, \quad 41, \quad 69-81$, doi:10.1016/j.ndteint.2007.09.001.

2. Abouhamad, M.; Dawood, T.; Jabri, A.; Alsharqawi, 
M.; Zayed, T. Corrosiveness mapping of bridge decks using image-based analysis of GPR data. Automation in Construction 2017, 80, 104-117, doi:10.1016/j.autcon.2017.03.004.

3. Francese, R.G.; Galgaro, A.; Grespan, A. Venice channel side-wall assessment with GPR techniquea case study. Journal of Applied Geophysics 2004, 56, 31-40, doi:10.1016/j.jappgeo.2004.03.001.

4. Evans, R.; Frost, M.; Stonecliffe-Jones, M.; Dixon, N. A Review of Pavement Assessment Using Ground Penetrating Radar (GPR). 2008, 11.

5. Solla, M.; Lagüela, S.; González-Jorge, H.; Arias, P. Approach to identify cracking in asphalt pavement using GPR and infrared thermographic methods: Preliminary findings. NDT \& E International 2014, 62, 55-65, doi:10.1016/j.ndteint.2013.11.006.

6. Du, Y.; Chen, J.; Han, Z.; Liu, W. A review on solutions for improving rutting resistance of asphalt pavement and test methods. Construction and Building Materials 2018, 168, 893-905, doi:10.1016/j.conbuildmat.2018.02.151.

7. Wang, W.; Wang, L.; Xiong, H.; Luo, R. A review and perspective for research on moisture damage in asphalt pavement induced by dynamic pore water pressure. Construction and Building Materials 2019, 204, 631-642, doi:10.1016/j.conbuildmat.2019.01.167.

8. Leng, Z.; Al-Qadi, I.L. An innovative method for measuring pavement dielectric constant using the extended CMP method with two air-coupled GPR systems. NDT \& E International 2014, 66, 90-98, doi:10.1016/j.ndteint.2014.05.002.

9. Hoegh, K.; Khazanovich, L.; Dai, S.; Yu, T. Evaluating asphalt concrete air void variation via GPR antenna array data. Case Studies in Nondestructive Testing and Evaluation 2015, 3, 27 33, doi:10.1016/j.csndt.2015.03.002.

10. Xu, B.; Ranjithan, S.R.; Kim, Y.R. New Relationships Between Falling Weight Deflectometer Deflections and Asphalt Pavement Layer Condition Indicators. Transportation Research Record 2002, 1806, 48-56, doi:10.3141/1806-06.

11. Carrera, V.; Partal, P.; García-Morales, M.; Gallegos, C.; Pérez-Lepe, A. Effect of processing on the rheological properties of poly-urethane/urea bituminous products. Fuel Processing Technology 2010, 91, 1139-1145, doi:10.1016/j.fuproc.2010.03.028. 